\title{
Actual Duration
}

National Cancer Institute

\section{Source}

National Cancer Institute. Actual Duration. NCI Thesaurus. Code C93499.

The period of time over which the event or activity actually occurs. 